REVISTA DE DERECHO UNED, NÚM. 9, 2011

\title{
PROTOCOLO Y ESTADO DE LA CIUDAD DEL VATICANO. ESTADO DE LA CUESTIÓN
}

\author{
ENRIQUe SOMAVILLA RodRÍGUEZ ${ }^{1}$
}

Resumen: Con el título de Protocolo y Estado de la Ciudad del Vaticano, estado de la cuestión, se ha querido desarrollar el contexto de una realidad histórica que supone la existencia permanente de la Santa Sede a través de los tiempos como sujeto con personalidad jurídica internacional, mediante el cual, la Iglesia actúa y participa en el concierto de las naciones, y por otra, la personalidad internacional del Estado de la Ciudad del Vaticano; aquélla donde se trata de la soberanía espiritual y ésta de la soberanía político-temporal. En los Pactos Lateranenses de 1929, entre Italia y la Santa Sede, Tratado político, Convención financiera y Concordato, éste fue sustituido por otro en 1984, más en concreto en el artículo 2 del propio Tratado político establece: «Italia reconoce la soberanía de la Santa Sede en el campo internacional como atributo inherente a su naturaleza, de acuerdo con su tradición y con las exigencias de su misión en el mundo». De esta manera, partiendo del concepto de protocolo y sus clases, se pretende penetrar en el inmenso mundo de las tradiciones y costumbres del Estado de la Ciudad del Vaticano, teniendo en cuenta los diversos aspectos que son necesarios para su mayor comprensión: qué entendemos al hablar de la Santa Sede o Sede Apostólica y su ceremonial; qué entendemos por Estado de la Ciudad del Vaticano y su protocolo.

Palabras clave: Protocolo, Vaticano, Santa Sede, Liturgia, Pactos Lateranenses.

${ }^{1}$ Es Doctor en Teología Dogmática. Licenciado en Estudios Eclesiásticos. Máster en Doctrina Social de la Iglesia. Máster en Protocolo y Relaciones Institucionales. Máster en Derecho de la Unión Europea y Doctorando en el Departamento de Historia del Derecho en la Facultad de Derecho de la UNED. 
Abstract: With the title of Protocol and State of Vatican City, state of affairs, we tried to develop the context of a historical reality that is the continued existence of the Holy See through the ages as a subject of international legal personality by which the Church operates and participates in the concert of nations, and secondly, the international personality of the State of Vatican City is one where the spiritual sovereignty of sovereignty and this political storm. In the 1929 Lateran Treaty between Italy and the Holy See, Political Treatise, financial and Concordat Convention, he was replaced by another in 1984, more specifically in Article 2 of the Treaty itself political states: "Italy recognizes the sovereignty of the Holy See in the international arena as an attribute inherent in its nature, according to their tradition and the demands of his mission in the world. Thus, from the concept of protocol and its classes, is to enter the vast world of traditions and customs of the State of Vatican City, taking into account the various aspects that are necessary for better understanding: what we understand speak of the Holy See or the Apostolic See and ceremonial; what we mean by the State of Vatican City and its p With the title of Protocol and State of Vatican City, state of affairs, we tried to develop the context of a historical reality that is the continued existence of the Holy See through the ages as a subject of international legal personality by which the Church operates and participates in the concert of nations, and secondly, the international personality of the State of Vatican City is one where the spiritual sovereignty of sovereignty and this political storm. In the 1929 Lateran Treaty between Italy and the Holy See, Political Treatise, financial and Concordat Convention, he was replaced by another in 1984, more specifically in Article 2 of the Treaty itself political states: Italy recognizes the sovereignty of the Holy See in the international arena as an attribute inherent in its nature, according to their tradition and the demands of his mission in the world. Thus, from the concept of protocol and its classes, is to enter the vast world of traditions and customs of the State of Vatican City, taking into account the various aspects that are necessary for better understanding: what we understand speak of the Holy See or the Apostolic See and ceremonial; what we mean by the State of Vatican City and its protocol.

Key words: Protocol; The Vatican; The Holy See; Liturgy; Lateran Pacts.

Sumario: I. Introducción al concepto de Protocolo.-I.1. Protocolo y su definición: terminología.-I.2. Qué entendemos por Protocolo: sus clases.-II. Qué entendemos por la Santa Sede.-III. Protocolo de la Sede Apostólica o Santa Sede.-IV. Qué entendemos por 
Estado de la Ciudad del Vaticano.-V. Protocolo del Estado de la Ciudad del Vaticano.-VI. Conclusión.

\section{INTRODUCCIÓN AL CONCEPTO DE PROTOCOLO}

Podemos comenzar este ambicioso proyecto mediante el desarrollo del concepto a partir de lo que podemos entender por la palabra Protocolo y sus antecedentes, los diversos planteamientos que podemos acentuar, así como para situarnos realmente en el campo que vamos a trabajar y conocer el amplio panorama que tenemos delante de nuestro entorno social, político, económico, empresarial, deportivo, universitario y religioso. Comenzamos por lo que nos puede expresar la palabra Protocolo. Protocolo proviene del latín protocollum, que a su vez deriva del griego clásico protókolon, que significa «la primera hoja o tapa, encolada, de un manuscrito importante, con notas sobre su contenido» ${ }^{2}$. Existen dificultades en cuanto a la posible confusión que existe en torno a la palabra protocolo y ceremonial. Este último muy utilizado en Argentina. Aunque ambos son distintos, en la práctica se utilizan como sinónimos.

Pero lo que es válido para los países latinoamericanos no sirve para los europeos, dado que se utilizan de forma distinta, siendo éstos los que utilizan el primer término como protocolo ${ }^{3}$. El primer código conocido en la historia de la humanidad es el de Hammurabi, datado aproximadamente en el año $1750 \mathrm{a}$. de C. escrito sobre caracteres cuneiformes sobre una estela de basalto negro. Se trata de un código integral donde se recogían los criterios que regían los usos y costumbres de los habitantes de la primitiva Babilonia. En él se desarrollaban varios aspectos para tener en cuenta: ceremonia de coronación del rey con un lenguaje muy preciso y eficaz; de otra las precedencias a todos los niveles, con la especificación de cargos y de orden corporativo y de los grupos sociales en que se clasificaban su población; y por último las recomendaciones dadas a los funcionarios para que traten con cortesía y gran respeto a sus ciudadanos, sea cual fuere su status social y pongan el mayor cuidado en llevar y cuidar con esmero todas las celebraciones, actos y ceremonias del reino ${ }^{4}$. Siempre es bueno tener en cuenta las condiciones y las propias ma-

\footnotetext{
2 De Urbina, J. A., El gran libro de Protocolo, Madrid 2001, pág. 27. Fernández, F., Protocolo para todos. Práctica, secretos y anécdotas, Madrid 2010, pág. 17.

3 Vid. Fuente Lafuente, C., Protocolo oficial. Las instituciones españolas del Estado y su ceremonial, Madrid 2008, págs. 37-38.

${ }^{4}$ Vid. De Urbina, J. A., El gran libro de Protocolo, Madrid 2001, págs. 28-29.
} 
neras de llevar adelante sus estilos y formas, de lo constituido tanto como norma como costumbre según los criterios y usos adoptados por cada sociedad ${ }^{5}$. Podemos decir que el carácter, los medios y los fines como el objetivo final de lo que denominamos protocolo lo podemos establecer en los siguientes aspectos: el protocolo se basa en la cortesía, que es la esencia de la educación, que significa ante todo el respeto a las personas, más allá de su condición social, lengua, raza o religión. Por tanto respeto total a la persona humana sin importar sus características propias: qué haga, qué sea y dónde se encuentre. El fin del protocolo es que todas aquellas actividades en las que interviene $o$ dirige se hagan lo mejor posible, se hagan bien, y con la máxima dignidad. El protocolo está para resolver los problemas que puedan aparecer y nunca para crearlos. Debemos tener en cuenta que estas acciones o actividades en las que tiene una intervención directa el protocolo, constituyen una parte muy importante de la estructura y la acción de un Estado de Derecho, mejorar y perfeccionar la paz, el entendimiento y la prosperidad de una sociedad. Se trata de mejorar en todo lo posible la convivencia social de las personas y el propio protocolo insta a la mejora de dicha convivencia ${ }^{6}$.

\section{I.1. Protocolo y su definición: terminología}

Fue Constantino I, el Grande (272-337), el último de los grandes emperadores de Roma, el que otorgó la libertad de culto en el 313 en lo que se llamó el Edicto de Milán de 13 de junio, llevó a la Paz Constantiniana ${ }^{7}$; pero el cristianismo no pasaría a ser religión oficial del Imperio Romano hasta el emperador Teodosio (347-395) en el 380 con el Edicto de Tesalónica el 28 de febrero 8 ; tras 67 años de libertad de cultos y que al establecer el cristianismo como religión oficial del Estado, todo el Imperio Romano pasaba a ser cristiano, marcó la frontera entre lo que entendemos como «protocolo civil, laico o aconfesional del Estado» y el «protocolo religioso o liturgia» de la religión cristiana, que pasaría a ser más tarde, oficial del Estado. Es a partir

${ }^{5}$ Evidentemente no es igual una sociedad antigua que medieval, una moderna que contemporánea. Tampoco se puede establecer las mismas exigencias para Oriente que para Occidente. Siempre habrá que tener muy en cuenta su propia identidad e idiosincrasia. Por ejemplo: el luto en Japón es el blanco y occidente es negro.

6 Vid. De Urbina, J. A., El gran libro de Protocolo, Madrid 2001, págs. 29-30.

7 Vid. Reina, V.-Reina, A., Lecciones de Derecho Eclesiástico español, Barcelona 1983, págs. 19-23.

8 Vid. Reina, V.-Reina, A., Lecciones de Derecho Eclesiástico español, Barcelona 1983, págs. 36-39. 
de este momento cuando el protocolo se hace más civil, perdiendo sus concomitancias religiosas y el religioso es incorporado a la liturgia cristiana. Con la caída del Imperio Romano, muchos aspectos desaparecerán para comenzar otros diversos.

Con el paso del tiempo las diversas Iglesias cristianas, incluida la católica, posee su verdadero protocolo, pero separado de la liturgia, aunque trabaje conjuntamente con ella ${ }^{9}$. Si viésemos las diversas acepciones sobre el concepto de protocolo hay tantas como diccionarios. Y si deseamos llegar a una conjunción de ideas y opiniones podemos establecer que protocolo es «el conjunto de normas o reglas establecidas por ley, decreto, disposiciones o costumbres, así como técnicas específicas tradicionales y modernas que son de aplicación para la organización de los actos públicos y privados de carácter formal ya sean de naturaleza oficial o no oficial, y que se ejecutan con solemnidad o sin ella»; ceremonial sería "el desarrollo y contenido de los actos, así como conjunto de formalidades para la celebración de los mismos»; por último se entiende por etiqueta «las reglas que se observan en el desarrollo de los actos públicos solemnes» ${ }^{10}$.

\section{I.2. Qué entendemos por Protocolo: sus clases}

Por Protocolo podemos también entender «aquella disciplina que, con realismo, técnica y arte, determina las estructuras o formas bajo las cuales se desarrolla una actividad humana pluripersonal e importante; con el objeto de su eficaz realización y, en último lugar, de mejorar la convivencia». O también: «Protocolo es aquella actividad que determina las formas bajo las cuales se han de llevar del mejor modo posible, las relaciones del ser humano con sus semejantes ${ }^{11}$. Podemos entender como Protocolo "la trascripción escrita de los usos, costumbres y tradiciones de un determinado país o territorio en fórmula reglamentadas». O también es «el ordenamiento reglado y jerarquizado de los concurrentes a un acto público o privado, según la naturaleza y fines del mismo, en razón del cargo que ostenten los invitados y de la causa de la presencia en torno al anfitrión» ${ }^{12}$.

9 Vid. De Urbina, J. A., El gran libro de Protocolo, Madrid 2001, págs. 30-31.

${ }^{10} \mathrm{Vid}$. Fuente Lafuente, C., Protocolo oficial. Las instituciones españolas del Estado y su ceremonial, Madrid 2008, págs. 41-42.

${ }_{11}$ De Urbina, J. A., El gran libro de Protocolo, Madrid 2001, pág. 33.

12 Vilarrubias, F. A., Tratado de Protocolo del Estado e Internacional, Oviedo 1994, pág. 21. 
Teniendo en cuenta todos estos principios, el ordenamiento protocolario debe contemplar el ámbito de cada acto, que ha de estar concretado en un determinado espacio, abierto o cerrado, teniendo en cuenta sus clases, ya sea civil, militar o religioso, y en función a quienes hayan de ocuparlo, bien por un tiempo determinado o para un uso concreto, con la finalidad de jerarquizar personas y cosas. Todo este ordenamiento posee sus propias características en función de si se conmemora un hecho histórico, de origen civil, militar o religioso, o bien desarrollar un acto oficial con carácter político, empresarial, deportivo, social o de exequias ${ }^{13}$. El hombre necesita, como animal social, vivir con otros en el seno de una comunidad organizada. Para ello es preciso que existan unas normas de conducta, aceptadas, asumidas y compartidas entre todos, de tal forma que puedan garantizar una adecuada y pacífica vinculación entre unos y otros.

En una sociedad acelerada y cambiante como la nuestra, las dudas acerca de la manera correcta de llevar a cabo nuestros comportamientos, y maneras de hacer lo que es preciso, de la mejor manera posible y de la forma más adecuada, es necesario que ciertas cuestiones se hagan bajo las condiciones de lugar, tiempo y circunstancias mediante el refuerzo y el impulso de los valores éticos de la persona. Ponernos de acuerdo con el significado de los signos y los símbolos que expresamos, la necesidad de un grado de cohesión en la misma sociedad y la participación activa entre todos sus miembros, supone un grado de formación y educación de la sociedad a la que pertenecen. Ni ha habido ni habrá nunca una civilización que no posea sus propios usos sociales, ceremoniales y modos de ser, pensar y de estar. El protocolo ha estado ceñido, en numerosas ocasiones a la dimensión religiosa del hombre. No solamente fue Egipto en su esplendor que unía la divinidad y el poder político en la persona del Faraón sino también en otras civilizaciones como en la corte persa con el rey Darío I, donde regía un ceremonial muy preciso. El acceso al propio rey suponía atravesar un protocolo ya establecido de diversas ceremonias y tenía como finalidad, entre otras, conseguir que los príncipes no apareciesen sin previo aviso.

Si vamos a la Sagrada Escritura ${ }^{14}$ nos encontramos muchos pasajes del AT con referencias a los aspectos protocolarios en donde los banquetes de algunos personajes son muy elocuentes: Nabucodono-

13 Vilarrubias, F. A., Tratado de Protocolo del Estado e Internacional, Oviedo 1994, págs. 21-22.

14 Vid. Lc 14, 7-11. 
sor $^{15}$, Salomón, Holofernes ${ }^{16}$, Saúl ${ }^{17}$, Gedeón ${ }^{18}$. Por otra parte aparece la Reina de Saba al hablar del rey Salomón ${ }^{19}$. También en el NT aparecen en los evangelistas cuando advierte que los escribas y fariseos, que gustan de los primeros puestos en los banquetes y de los primeros asientos en las Sinagogas ${ }^{20}$. Si hay algo que ha hecho evolucionar al hombre eso ha sido la educación. Desde los tiempos más remotos se ha tenido conocimiento de una serie de pautas sociales y normas de comportamiento. El ser educado no es una moda pasajera pues la buena educación abre puertas y hace posible el conocimiento entre las personas. Lo que en algunas culturas posee un determinado significado, para otras puede parecer algo totalmente extraño pero no por ello debemos de dejar de comportarnos siempre de un modo respetuoso. La actitud siempre abierta en toda clase de acontecimientos nos dará la oportunidad de actuar de la manera más correcta y oportuna.

El protocolo trata siempre de conseguir que no aparezcan distorsiones en cualquier tipo de acontecimientos o eventos con participantes de diversas culturas y procedencias. En el protocolo existe una fuerte componente de índole religiosa. Por último decir que entendemos por Protocolo, según el diccionario de la Real Academia Española la serie o conjunto de formalidades para cualquier acto público o solemne. Como acepción de Protocolo decimos que es la regla ceremonial, diplomática o palatina, establecida por decreto o por costumbre. Por tanto el Protocolo reconoce las jerarquías del ordenamiento institucional y reconoce el rango de las autoridades y sus respectivas precedencias, preeminencias y primacías.

\section{QUÉ ENTENDEMOS POR LA SANTA SEDE}

La Santa Sede o Sede Apostólica es la expresión con que se alude a la jerarquía de Papa en tanto en cuanto es la cabeza Suprema de la Iglesia católica, en distancia a lo que denominamos Estado Ciudad del Vaticano que es un Estado soberano, aunque ambas realidades están profundamente relacionadas y hecho es que el Vaticano como Estado, existe al servicio de la Santa Sede y a la Iglesia. La Santa

15 Vid. Dn 1, 3-5.

16 Vid. Jdt $12,1-3$ y $12,10-12$.

17 Vid. 1 S 9, 22-24.

18 Vid. Jc 7, 5-7.

19 Vid. 1 R 10,1-13.

20 Vid. Mt 23, 6-7; Mc 12, 38-40; Lc 20,46-47; Lc 11, 43. 
Sede posee personalidad jurídica propia y en estricto rigor es la que mantiene relaciones diplomáticas con los diversos países del mundo. Los llamados Pactos Lateranenses o de otra forma Pactos de Letrán de 11 de febrero de 1929, establecieron el origen del Estado de la Ciudad del Vaticano y al Concordato entre Italia y la Santa Sede y fueron ratificados por el Reino de Italia y la Santa Sede, poniendo fin a las disputas sobre la llamada Cuestión Romana. De una parte la pérdida de los Estados Pontificios y de otra Italia reconocía la soberanía absoluta de dicho Estado Vaticano. Las negociaciones se llevaron a cabo por parte de la Iglesia del cardenal Secretario de Estado Pietro Gasparri y de parte de Italia por su primer ministro Benito Mussolini. Los acuerdos fueron ratificados solemnemente por ambas partes el 7 de junio de 1929. Entre todos los organismos de la Santa Sede prestan servicio 2732 personas, de las cuales son 761 eclesiásticos, 334 religiosos y 1637 laicos $^{21}$. Su alusión como origen del término viene aparejada al Santo Padre y a su autoridad pontificia, como Obispo de Roma, sucesor de Pedro y Cabeza suprema de la Iglesia católica. De ahí el término utilizado de Sede Apostólica o Santa Sede.

Las sedes patriarcales desde el principio fueron Roma, Constantinopla, Antioquía, Alejandría y Jerusalén. Se las conocía y tenían esta denominación porque desde el principio eran sedes apostólicas. Tras la abdicación de Diocleciano en el 305, violentas luchas habían enfrentado a sus sucesores; en el 325, Constantino I el Grande, se convirtió en el único soberano del Imperio, cuya unidad se vio de nuevo comprometida a su muerte, en 337: sus tres hijos y sus dos sobrinos, sin contar con sus hermanastros, compitieron para sucederle. El mundo romano, próximo a su fin, se hallaba dividido entre dos imperios y dos sistemas religiosos. Antes tomó unas de las decisiones geopolíticas más importantes llevadas a cabo por Constantino: fue el traslado el 11 de mayo del año 330, la capitalidad del imperio romano hacia la nueva Roma. La ciudad de Bizancio, en la zona más oriental del mediterráneo fue la elegida, pasando a llamarse Constantinopla (actual Estambul), en honor del emperador. Este cambio favoreció ostensiblemente a la parte oriental o griega de su imperio ya que fue transformándose en el centro religioso, social y político del mundo cristiano de Oriente. Esto propició una mayor división entre la Iglesia católica, tanto por el lenguaje como por la geografía. La Iglesia de Roma de habla latina, en Occidente, y la Iglesia de Constantinopla, de habla griega, en Oriente se distanciaban aún más. En el siglo $\mathrm{V}$ fueron frecuentes las disputas que, por razones de supre-

21 Vid. Annuario Pontificio, Ciudad del Vaticano 2011, 2347 págs. 
macía, sostuvieron entre sí los obispos de las tres grandes ciudades del Imperio romano: Constantinopla, Alejandría y Roma. La primera, Constantinopla, basaba sus pretensiones en el hecho de ser la capital del imperio. La segunda, Alejandría, por ser el centro cultural y comercial. La tercera, Roma, ofrecía su pasado glorioso y los recuerdos. A ellas se uniría, definitivamente, Antioquía y Jerusalén y quedarían como las cinco grandes sedes patriarcales de la Iglesia de Cristo, pero Roma quedaba emplazada como sede del Sucesor de san Pedro y como cabeza del Colegio Apostólico.

\section{PROTOCOLO DE LA SEDE APOSTÓLICA O SANTA SEDE}

La aplicación del concepto de Protocolo a la Sede Apostólica será diverso de lo que se establece para la Iglesia y también distinto para el Estado Ciudad del Vaticano. El protocolo de la Santa Sede se refiere fundamentalmente a los actos referidos al Santo Padre en sus funciones ministeriales, como Obispo de Roma, sucesor del Príncipe de los Apóstoles, Vicario de Cristo y Siervo de los siervos de Dios. Por tanto, son todas las actuaciones y actividades que mantiene en función de dichas responsabilidades. También se encuentran dentro de dicho protocolo las actividades de la Secretaría de Estado y el conjunto de la Curía Romana. Todas las acciones litúrgicas pertenecen al Pueblo de Dios. De ahí derivan tanto el respeto como la atención debida y que se debe prestar en todo momento a cada persona, puesto que los fieles cristianos tienen derechos y deberes como miembros de la asamblea eclesial que deben ser siempre respetados. Esto no suele ser fácil dado que se trata de temas muy troncales y que al final siempre miran a la Sede Apostólica porque en ocasiones se desconocen las oportunas determinaciones dadas por ella. Es primordial conocer los ritos y los gestos de la renovada liturgia, para que su ejecución no quede al subjetivismo particular de cada cual; de esta manera podemos llegar a tener criterios teológicos adecuados, oportunos, claros y auténticos.

Estas acciones litúrgicas, tan vitales para la esencia de la Iglesia, por medio de los documentos posconciliares, se instruyen en la verdad y la unidad y así poder expresar la santidad de los signos y alcanzar una celebración más plena, más activa y más comunitaria. La Santa Sede en todas las celebraciones, actos y ceremonias está llena de una viva liturgia. Porque la liturgia es belleza y dignidad, es orden y disposición, es ante todo una escuela de vida. Desde la liturgia podemos también ir aprendiendo que la vida es un don, algo inespera- 
do, regalado, tesoro, sin contrapartida alguna, que es de Dios, que de Dios viene y a Dios vuelve. Aquí entramos en el terreno de la fe y la virtud teologal de la fe también es algo otorgado, entregado, dado por Dios y sólo podemos concluir que se trata de un misterio, inaccesible si no es a través de esa fe; que somos miembros del cuerpo de Cristo que es la Iglesia y que el misterio de la presencia de Jesucristo es fuente inagotable de esperanza. Esto, y no otra cosa, es lo que la Santa Sede, trata de trasmitir en todas sus celebraciones: aquellas que dimanan de la actividad pastoral del Santo Padre y del resto de la $\mathrm{Cu}-$ ria Romana, en cuanto que es parte de la Sede Apostólica como hemos dicho anteriormente. Por eso la liturgia se entremezcla en el protocolo, como expresiones o maneras de proceder y que se han de seguir en los actos que realiza el Papa u otras autoridades que le representan; pero dichos actos suelen ser bien distintos. Por eso la importancia que tiene, que posee y expresa la liturgia a la hora de plasmar y vivir estos acontecimientos tan relevantes ${ }^{22}$. Siempre la Sede Apostólica ha velado por los detalles de manera rigurosa a la hora de establecer los protocolos adecuados para cada acto o celebración particular o concreta.

\section{QUÉ ENTENDEMOS POR ESTADO DE LA CIUDAD DEL VATICANO}

Ya hemos definido qué entendemos por Iglesia y qué entendemos por la Santa Sede. Iglesia y Estado Ciudad del Vaticano son dos cosas perfectamente distintas, aunque resulten estar íntimamente unidas. Se trata de un Estado, un organismo político y soberano. La existencia de un Estado implica un poder político sobre una ciudadanía y sobre un territorio determinado que posee la finalidad de conseguirles la consecución de un bienestar en el orden temporal mediante el desarrollo total de su personalidad. Además del territorio y la población necesita una autoridad; dicha autoridad con respecto al orden interno manifiesta realmente una supremacía que se enmarca en la soberanía nacional y con respecto al orden externo, implica una independencia hacia los demás Estados soberanos. En definitiva, el Estado Ciudad del Vaticano supone un poder político independiente, que mantiene relación con terceros países, soberano sobre un determinado territorio que se encuentra entre las murallas leoninas, la Basílica y la Plaza de san Pedro que abarca la columnata de Bernini y

22 Vid. Pardo Rodríguez, A., Documentación Litúrgica posconciliar. Enchiridion, Barcelona $2000^{4}, 1586$ págs. 
la línea de mármol blanco que cierra los brazos incompletos de la misma. Además de las otras Basílicas, otros edificios llamados extraterritoriales que se encuentran repartidos por la ciudad de Roma y la villa pontificia de Catelgandolfo, residencia veraniega del Santo Padre. El soberano del Estado Ciudad del Vaticano es el Papa ${ }^{23}$. Como autoridad suprema del Estado Ciudad del Vaticano, el Santo Padre ejerce esa autoridad temporal y política y está en función del servicio de la autoridad espiritual que tiene y que la ejerce como Jefe supremo de la Iglesia. Si su poder espiritual se extiende por todo el orbe católico, su poder temporal queda reducido a su minúsculo territorio ${ }^{24}$.

La Iglesia mantiene su carácter supranacional, puesto que el Santo Padre no puede quedar sujeto a ninguna autoridad civil o política. Incluso cuando la Santa Sede no dispuso de ese territorio, desde la pérdida de los Estados Pontificios en 1870, tras la unidad italiana ${ }^{25}$ hasta la firma de los Tratados Lateranenses en 1929, aún sin territorio definido no era un Estado, seguía siendo una potencia y siempre mantuvo ese prestigio y reconocimiento internacional. Incluso se puede decir que fue en ese momento histórico cuando la Sede Apostólica obtenía la consagración y el reconocimiento de la soberanía espiritual en la práctica y en la vida internacional ${ }^{26}$. De aquí que la soberanía y la independencia en el concierto de las naciones, se le reconoce esa personalidad jurídica internacional, incluso por los Estados no católicos ${ }^{27}$. De aquí que soberanía temporal y espiritual son dos realidades bien distintas, pero la soberanía temporal es el soporte y el punto de apoyo indispensable para el ejercicio con libertad del poder espiritual ${ }^{28}$. Pero también es preciso argumentar que el ejercicio de ese poder espiritual que ejerce la Iglesia y que lo hace sobre toda la cristiandad, no se podría realizar con verdadera independencia si el Santo Padre no posee un territorio sobre el que puede ejercer su soberanía de manera totalmente libre. En resumen, un territorio

${ }^{23}$ Vid. Martín Martínez, I., Sobre la Iglesia y el Estado, Madrid 1989, págs. 272273.

${ }^{24}$ Vid. L. PÉrez Mier, L., Iglesia y Estado nuevo. Los concordatos ante el moderno Derecho Público, Madrid 1940, págs. 190-194: especialmente 191-192; MARTín MARTíNEZ, I., Sobre la Iglesia y el Estado, Madrid 1989, pág. 272.

${ }_{25}$ Vid. Palacio Atard, V., Manual de Historia Universal, Madrid 1960, págs. 196204. Especialmente págs. 202-204.

${ }^{26}$ Vid. Pérez Mier, L., Iglesia y Estado nuevo. Los concordatos ante el moderno Derecho Público, Madrid 1940, págs. 194-195. 277.

27 Vid. Martín Martínez, I., Sobre la Iglesia y el Estado, Madrid 1989, págs. 276-

28 Vid. Pérez Mier, L., Iglesia y Estado nuevo. Los concordatos ante el moderno Derecho Público, Madrid 1940, págs. 198-199. 
que constituye un Estado sobre el cual se asiente la Sede Apostólica y que sea la garantía de la independencia espiritual de la Iglesia. De no ser así, podía estar mediatizado en el ejercicio de sus poderes y sus acciones podían verse coaccionadas y dificultadas en gran medida. Durante algunas épocas no existió como en los tres primeros siglos o desde 1870 a 1929 y a pesar de ello no dejó de existir. Pero siempre es mejor que exista para salvaguardar y garantizar mediante la existencia de ese territorio en el que es único soberano Su Santidad. Esta es la justificación de la existencia del actual Estado Ciudad del Vaticano ${ }^{29}$.

Por tanto, la Iglesia católica es, desde el punto de vista jurídico, una comunidad autónoma e independiente de cualquier potestad humana, es decir, soberana, el ejercicio de la soberanía, que es espiritual y que siempre corresponde al Romano Pontífice. Para su ejercicio necesita el Papa la colaboración del conjunto de los diversos organismos, subordinados a su persona. A este conjunto, a cuya cabeza se encuentra el mismo Santo Padre lo denominamos Santa Sede o Sede Apostólica. De esta manera ante la comunidad eclesial y su ordenamiento, la Santa Sede es el órgano supremo de dirección de la Iglesia, bien sea el Papa, la Secretaría de Estado y otras instituciones de la Curia Romana. Se puede decir que la Sede Apostólica viene a ser la suprema dirección y representación tanto de la Iglesia como del Estado Ciudad del Vaticano; uno lleva el sentido místico, otro el político-estatal $^{30}$. Para llegar a esta nueva realidad llevó un largo período de tiempo, más bien largo, que se había iniciado en agosto y finalizaron en octubre de 1926. Se trataba de un inicio de las negociaciones y comenzarían oficialmente el 6 de octubre de dicho año. El día 24 se fijaron las bases de negociación. Éstas siguieron con altibajos durante 1927-1928. A partir de noviembre de 1928 las negociaciones entraron en su fase final aunque hubo que retrasarlas debido al fallecimiento del Delegado italiano Domenico Barone.

A partir de ese momento las negociaciones se llevaron a cabo a través de Benito Mussolini y Pío XI por medio de Eugenio Pacelli, bajo la atenta mirada del cardenal Secretario de Estado Pietro Gasparri. Los acuerdos se firmaron el día 11 de febrero de 1929 en el Palacio de Letrán: Se conformaron tres acuerdos: en primer lugar el Tratado político, que ponía fin a la Cuestión Romana, por la que se

29 Vid. Martín MartíneZ, I., Sobre la Iglesia y el Estado, Madrid 1989, pág. 277.

30 Vid. Corral Salvador, C., Derecho internacional concordatario, Madrid 2009 (=BAC 684), págs. 93-95; También CORRAL SALVADOR, C., La relación entre la Iglesia y la comunidad política, Madrid 2003 (=BAC 633), págs. 291-307. Especialmente págs. 297305. 
creaba el Estado Ciudad del Vaticano, bajo la soberanía del Santo Padre y el reconocimiento del Reino de Italia con la dinastía de los Saboya y teniendo a Roma como su capital; en segundo lugar el Convenio financiero que establecía la indemnización por parte de Italia a la Santa Sede por los territorios a los que admitía su renuncia; en tercer lugar al Concordato entre Italia y la Santa Sede mediante el cual se regulaban las relaciones entre la Iglesia y el Estado italiano ${ }^{31}$. Como anejos al Tratado político se encontraban la delimitación del territorio del Estado Ciudad del Vaticano; la relación de doce inmuebles vaticanos que gozan de extraterritorialidad ${ }^{32}$ y la exención de impuestos, y por último la relación pormenorizada de todos los inmuebles exentos de impuestos y de expropiación ${ }^{33}$.

El Estado Ciudad del Vaticano (SCV), quedó configurado definitivamente por el Tratado en sus líneas básicas y fue estructurado en seis leyes fundamentales que el mismo día del inicio o de su nacimiento en cuanto tal, promulgaba el Papa Pío XI y que son las siguientes: $1^{\circ}$ Ley Fundamental de la Ciudad del Vaticano, modificada por Motu Proprio de 1 de mayo de 1946; Motu Proprio de 28 de marzo de 1968; Ley L de 21 de mayo de 1969 y Quirógrafo de 6 de abril de 1984; al presente sustituida íntegramente por Juan Pablo II, por la Ley Fundamental del Estado Ciudad del Vaticano de 26 de noviembre de 2000, que a la vez abrogaba todas las normas vigentes en el Estado en contraste con la presente Ley y entró en vigor el 2 de febrero de 2001; $2^{\circ}$ Ley sobre las fuentes del Derecho, modificada por la Ley de 21 de junio de 1969 y el Motu proprio de 1 de mayo de 1946; $3^{\circ}$ Ley sobre la ciudadanía y la residencia, modificada por la Ley de 21 de junio de 1969; $4^{\circ}$ Ley sobre la organización administrativa, modificada por la Ley XXXII de 1 de diciembre de 1932, Ley LI de 24 de junio de 1969 y Quirógrafo de 6 de abril de 1984; $5^{\circ}$ Ley sobre la organización económica, comercial y profesional, modificada por la Ley de 21 de junio de 1969; $6^{\circ}$ Ley de Seguridad Pública ${ }^{34}$. La ratificación se llevó a

${ }^{31}$ El Concordato fue reformado tras unas conversaciones y largas negociaciones el 18 de febrero de 1984, en Villa Madama, sede representativa del Gobierno italiano; se firmó el Acuerdo entre la Santa Sede y la República Italiana, por el que se modificaba el Concordato Lateranense de 11 de febrero de 1929.

32 Vid. Corbellini, G., "Il Vaticano: territorio, aree esterne, istituzione», en Governatorato dello Stato della Città del Vaticano, Lo Stato della Città del Vaticano. Atti del convegno sugli 80 anni (12-14 febbrairo 2009), Città del Vaticano 2010, págs. 50-57.

33 Corral, C.-Urteaga, J. M., Diccionario de Derecho Canónico, Madrid 1989, voz VATICANO.

34 Corral Salvador, C., La relación entre la Iglesia y la comunidad política, Madrid 2003 (=BAC 633), págs. 324-326; Cf. Inter Sanctam Sedem et Italiae Regnum Con- 
cabo el 7 de junio de 1929, festividad del Sagrado Corazón de Jesús ${ }^{35}$. En 1984 se produjeron cambios para la acomodación de los Tratados Lateranenses debido al nuevo Concordato entre Italia y la Santa Sede $^{36}$. Por último en el año 2001 entró en vigor una modificación de la Ley Fundamental del Estado Ciudad del Vaticano, otorgada por Juan Pablo II $^{37}$.

El Estado Ciudad del Vaticano posee su bandera, sello y escudo, como lo prescribe la Ley Fundamental: «La bandera de la Ciudad del Vaticano se compone de dos campos divididos verticalmente; uno de color amarillo, del lado del asta y blanco el del otro lado. Este último llevará la tiara y las llaves. El Escudo representará la tiara con las llaves sobre fondo rojo; el sello llevará en el centro la tiara y las llaves y alrededor las palabras Stato Città del Vaticano» (Art. 19). Las dimensiones de la bandera son 1:1. Como hemos dicho en la franja blanca figuran las dos llaves entrecruzadas, una de oro y la otra de plata, unidas con un cordón rojo o azul, y sobre las llaves aparece representada la tiara de los papas. Estos elementos también forman parte tanto del escudo del Vaticano como del blasón de cada pontífice. Las llaves entrecruzadas son las llaves de san Pedro. La llave amarilla además

VENCIONES. «Trattato fra la Santa Sede e 1 Italia», en AAS 21 (1929) 209-221; con el «Allegato I. Territorio dello Statu Della Cittá del Vaticano», en AAS 21 (1929) 223-226; con «Allegato II. Mobili con privilegio di extraterritorialità e con esenzione da espropriazioni e da tributi», en Acta Apostolica Sedis 21 (1929) 327-252; «Allegato III. Immobili esenti da espropriazioni e da tributi», en AAS 21 (1929) 253-271; "Allegato IV. Convenzione Financiaría», en AAS 21 (1929) 273; «Concordato fea La Santa Sede e 1 Italia», en AAS 21 (1929) 275-295; «Chirograph», en AAS 21 (1929) 297-306; «Post Mutuo Tradita Instrumenta Ratihabitionis Conventionum inter Apostolicam Sedem et Regnum Italiae», en AAS 21 (1929) 307. Acta Apostolica Sedis en adelante AAS. 296.

35 Vid. Martín Martínez, I., Sobre la Iglesia y el Estado, Madrid 1989, págs. 293-

36 Vid. Inter Sanctam Sedem et Italiam Coventiones, en AAS 77 (1984) 521-578; «Accordo tra la Santa Sede e la Republica Italiana che apporta modificazione al Concordato Lateranense», en AAS 77 (1984) 521-531; «Prottocolo Addizionale», en AAS 77 (1984) 532-535; «Prottocolo di aprovazione delle norme per la disciplina della materia di cui all art. $7 \mathrm{n}^{\circ} 6$ dell Accordo tra Santa Sede e la Republica Italiana che apporta modificazioni al Concordato lateranense», en AAS 77 (1984) 536-537; «Allegato Scambio di lettere...», en AAS 77 (1984) 538-546; «Norme circa gli enti e beni ecclesiastici in Italia...», en AAS 77 (1984) 547-578.

37 Vid. JuAn PaBlo II, «Legge Fondamentale dello Stato della Città del Vaticano», en AAS 92 (2000) 1054-1064; JuAn PaBlo II, Ley fundamental del Estado de la Ciudad del Vaticano del 26-11-2000, en AAS 92 (2000) 1054-1061 (74-80), Suplemento (01-022001) Anexo A, en AAS 92 (2000)1062 (81); Anexo B, en AAS 92 (2000)1063 (82); Anexo $C$, en AAS 92 (2000)1064 (83); G. CoRbELLINI, «Il Vaticano: territorio, aree esterne, istituzione», en Governatorato dello Stato della CitTÀ DEl Vaticano, Lo Stato della Città del Vaticano. Atti del convegno sugli 80 anni (12-14 febbrairo 2009), Città del Vaticano 2010, págs. 77-90. 
representa al poder espiritual de la Iglesia católica y la gris su poder temporal. El cordón es el símbolo del vínculo entre los dos poderes.

Las llaves son, desde el siglo XIV, símbolo de la Santa Sede. La tiara está compuesta por tres coronas que representan las atribuciones del papa como «pastor», «maestro» $\mathrm{y}$ «sumo sacerdote» de la Iglesia. La bandera antes de 1808 era roja y amarilla, los colores tradicionales del Senado y del Pueblo romanos, tras ser absorbidos por los ejércitos de Napoleón ${ }^{38}$.

Hay que tener en cuenta que los ataques militares y las dificultades políticas más importantes fueron a través de la historia, los de Napoleón, Garibaldi y Cavour que condujeron a la pérdida progresiva de los Estados Pontificios en 1870. En los apéndices se puede apreciar tanto la Bandera como el Escudo y el sello ${ }^{39}$. De esta manera, Estado, es como se designa oficialmente la Ciudad del Vaticano y como tal es reconocida por Italia en el Tratado (Art. 26) (40 $^{4}$ De igual manera ocurre en los demás organismos Internacionales ${ }^{41}$ en los que la Santa Sede es reconocida como tal a través de la historia, el derecho, la teología y que mantiene relaciones diplomáticas.

Por tanto, el ordenamiento constitucional vaticano queda enmarcado dentro de la Ley Fundamental de 1929, las modificaciones posteriores introducidas por Pío XII, de 20 de marzo de 1939, y el Motu Proprio "Con la legge» de 1 de mayo de 1946; la obra reformadora de Pablo VI con el Motu Proprio de 28 de marzo de 1968 y la nueva ley sobre el Gobierno del Estado Ciudad del Vaticano de 24 de junio de 1969; por último la reforma de Juan Pablo II, con la promulgación de la nueva Ley Fundamental de 26 de noviembre de 2000 y que entró en vigor el 22 de febrero de $2001^{42}$. Es imprescindible tratar de las fuen-

${ }^{38} \mathrm{Vid}$. M. A. Díaz Bonilla, M. A., Organización, ceremonial y protocolo en la Iglesia católica, Madrid 2002, págs. 125-128.

39 Vid. Juan Pablo II, «Legge Fondamentale dello Stato della Città del Vaticano», en AAS 92 (2000) 1054-1064; JuAn PABLo II, Ley fundamental del Estado de la Ciudad del Vaticano del 26-11-2000, en AAS 92 (2000) 1054-1061 (74-80), Suplemento (01-022001) Anexo A, en AAS 92 (2000)1062 (81); Anexo B, en AAS 92 (2000)1063 (82); Anexo C, en AAS 92 (2000)1064 (83);

40 C. CoRral SAlvador, La relación entre la Iglesia y la comunidad política, Madrid 2003 (=BAC 633), pág. 326.

41 Vid. G. CorbellinI, G., «Lo Stato della Città del Vaticano nell ambito internazionale», en Governatorato dello Stato della Città del Vaticano, Lo Stato della Città del Vaticano. Atti del convegno sugli 80 anni (12-14 febbrairo 2009), Città del Vaticano 2010, págs. 104-110.

42 Vid. Dalla Torre, G., «L Ordinamento costituzionale vaticano nel suo sviluppo storico», en Cammeo, F., Ordinamento giuridico dello Stato della Città del Vaticano, Città del Vaticano 2005, págs. 483-517. 
tes normativas y la función legislativa ${ }^{43}$. Es realmente muy importante el funcionamiento y el desarrollo de la actividad de Gobierno de la Ciudad y la organización administrativa ${ }^{44}$. De igual categoría y decisivo también es el ordenamiento judicial del Estado Ciudad del Vaticano ${ }^{45}$. El Santo Padre ostenta los tres poderes representativos del Estado. El poder ejecutivo, el legislativo y el judicial. El primero se ejerce mediante el Presidente de la Comisión, del secretario y vicesecretario generales. Para temas decisivos es necesario la aprobación de la Comisión y de acuerdo con la Secretaria de Estado ${ }^{46}$; el segundo es ejercido por una Comisión de cardenales que está presidida por el cardenal-Presidente. Para los proyectos de ley se tiene en cuenta a los consejeros de Estado, expertos y otros organismos dependientes de la Sede Apostólica o del Estado ${ }^{47}$; para el tercero se ejerce en nombre del Santo Padre por los órganos pertinentes creados por el ordenamiento judicial del Estado Ciudad del Vaticano ${ }^{48}$. En resumen, todo queda en manos del Papa.

\section{PROTOCOLO DEL ESTADO DE LA CIUDAD DEL VATICANO}

Ya hemos visto lo que podíamos entender por el protocolo en la Iglesia que se realiza especialmente por medio de la Liturgia; por el protocolo en la Santa Sede que se refiere esencialmente a los actos, celebraciones y ceremonias en las que participa el Santo Padre, en cuanto Vicario de Cristo, y de ahí tenemos que establecer las líneas del protocolo en el Estado de la Ciudad del Vaticano. Éstas se refieren esencialmente a los eventos y acontecimientos relacionados en torno

43 Vid. Bonnet, P. A., "Le fonti normative e la funzione legislativa», en CAmmEO, F., Ordinamento giuridico dello Stato della Città del Vaticano, Città del Vaticano 2005, págs. 519-566.

44 Vid. Marrone, G., "Attività di Governo e organizzazione amministrativa», en Cammeo, F., Ordinamento giuridico dello Stato della Città del Vaticano, Città del Vaticano 2005, págs. 567-594.

45 Vid. PICARDI, N., "Ordinamento Giudiziario dello Stato della Città del Vaticano», en Cammeo, F., Ordinamento giuridico dello Stato della Città del Vaticano, Città del Vaticano 2005, págs. 595-616; PRIETO, V., Relaciones Iglesia-Estado. La perspectiva del Derecho canónico, Salamanca 2005, págs. 229-233.

46 Vid. Juan Pablo II, Ley fundamental del Estado de la Ciudad del Vaticano del 2611-2000, en AAS 92 (2000) 1054-1061 (74-80), Suplemento (01-02-2001): Cf. art. 5 y 6 y se desarrollan sus funciones en los arts. 9 y 10.

47 Vid. JuAn Pablo II, Ley fundamental del Estado de la Ciudad del Vaticano del 2611-2000, en AAS 92 (2000) 1054-1061 (74-80), Suplemento (01-02-2001): Cf. art. 4, 2.

48 Vid. JuAn PaBlo II, Ley fundamental del Estado de la Ciudad del Vaticano del 2611-2000, en AAS 92 (2000) 1054-1061 (74-80), Suplemento (01-02-2001): Cf. art. 15 y 16. 
a la figura del Papa en cuanto Jefe del Estado Ciudad del Vaticano, como pueden ser las Visitas de Estado de los Jefes de Estado o de Gobierno de cualquier país en visita oficial; de las presentaciones de Cartas Credenciales de los nuevos embajadores, acreditados cerca de la Santa Sede.

También entran dentro de este marco, el estricto protocolo que se sigue ante la enfermedad grave del Santo Padre y posterior fallecimiento del Romano Pontífice: pasos que se deben dar; funcionamiento de las previsiones sucesorias; organización por el Consejo de cardenales de la Sede vacante según la normativa prevista; actuación de cardenal Camarlengo; certificación de la muerte; comunicación oficial a toda la Iglesia del luctuoso suceso; signos elocuentes del hecho como el toque de difuntos de las campanas de la Basílica Vaticana; exposición pública de los restos mortales en la Basílica de san Pedro; decisión sobre las fechas para las reuniones de las Congregaciones Generales; fecha para el Funeral oficial en la Plaza de san Pedro con la asistencia de las autoridades italianas y del resto del mundo; Celebración del Solemne funeral y posterior inhumación en las grutas vaticanas; sistema de elección del nuevo Papa; disposiciones para la convocatoria del Cónclave; elaboración de Informes sobre la situación, a día de la fecha, de la Santa Sede y de la Iglesia; normas establecidas sobre la Elección del nuevo Obispo de Roma; Missa pro eligendo Papa entre 15 y 20 días a partir de fallecimiento Sumo Pontífice; los cardenales se reúnen en la Capilla Paulina por la tarde de ese día y van en procesión solemne a la Capilla Sixtina; juramento de cumplir fielmente el encargo y de guardar secreto según las normas establecidas; el Maestro de las Celebraciones litúrgicas pronuncia Extra omnes y todos salen de la Capilla, excepto los Cardenales; comunicaciones del resultado de las votaciones realizadas en la Capilla Sixtina; las fumatas negras y la blanca; los redobles de las Campanas de la Basílica que saludan al recién elegido para la cátedra de san Pedro; el paso a la sala del llanto; el beso y abrazo de todos los cardenales al nuevo Vicario de Cristo en señal de obediencia y sumisión; la salida a la Logia Central de la Basílica para el saludo del Santo Padre y la Bendición a la urbe y al orbe; preparación de la ceremonia de entronización que actualmente se denomina celebración del inicio de pontificado o también Misa solemne para el inicio del ministerio pastoral del Santo Padre; anuncio de dicha celebración; solemne celebración eucarística con la presencia de autoridades y delegaciones internacionales llegadas para tal acontecimiento y saludo oficial de dichos representantes al Santo Padre en el interior de la Basílica, finalizada la ceremonia religiosa. 
Por tanto, de una parte, aparecen aquellos actos protocolarios que tienen íntima conexión con las labores de Estado, aunque hayan sido delegadas anteriormente a las distintas áreas reservadas de la Administración y la representación de los tres poderes del Estado, como pueden ser los relativos a los aspectos diplomáticos; de otra se encuentran las relativas a la persona del Papa en cuanto persona. Podemos observar la cantidad de detalles y aspectos que hay que tener en cuenta; así como la entidad de los mismos, como resultado de la misma esencia de lo que representa la Iglesia, como pueblo de Dios, que peregrina en la tierra; la supremacía de la Sede Apostólica que viene impuesta en función de la íntima relación y la estrecha vinculación e intercomunicación constante y fehaciente con la figura del Papa; con la existencia del Estado Ciudad del Vaticano que como cualquier otro Estado posee sus divisas como tal, para acrecentar, mantener y expresar la libertad de acción que debe poseer en todo momento el Jefe del Estado Ciudad del Vaticano y la no concurrencia de cualquier otro poder temporal que pueda situarse por encima de toda actuación pontificia, para el mejor desarrollo de la misión evangelizadora de la Iglesia y de la potestad del Romano Pontífice en su compromiso de anunciar el Reino de Dios.

Entre otros aspectos se pueden tener en cuenta dos detalles como botón de muestra en el protocolo del Estado de la Ciudad del Vaticano. En el primer caso se trata del protocolo con respecto a Italia que según los Pactos Lateranenses, sitúan en el protocolo estricto a los cardenales asistentes a un evento, inmediatamente detrás del Presidente de la República y de los Presidentes de las Cámaras legislativas recibiendo los honores establecidos. Cuando se trata de la asistencia de los obispos que acuden a distintos actos públicos, éstos van siempre detrás de las autoridades civiles provinciales italianas. En el segundo caso, en algunas ocasiones las audiencias oficiales del Papa a los Jefes de Estado o de Gobierno, que se celebran habitualmente en la Biblioteca privada del Santo Padre en el Palacio Apostólico, se introducen cambios en el protocolo ya establecido, y se llevan a cabo en otras dependencias del Estado Ciudad del Vaticano, como la Torre de san $\mathrm{Juan}^{49}$, que se alza junto a las Murallas de la Ciudad Leonina, y que se encuentra en el interior de la misma, en medio de amplios, frondosos y bien cuidados jardines.

Las relaciones entre Italia y la Santa Sede, por sus características particulares siempre han sido muy consideradas. Debido a todo el

49 Esto se produjo durante la visita del Presidente norteamericano George W. Bush al Papa Benedicto XVI el 9 de junio de 2007. Bush quiso despedirse oficialmente del Santo Padre al final de su mandato, antes de abandonar la Casa Blanca. 
proceso de la unificación italiana, la posterior Ley de Garantías ${ }^{50}$ de 1871, nunca aceptada por el Santo Padre, Pío IX; su famosa reclusión en el Vaticano hasta la normalización entre ambas sociedades, la civil y la eclesiástica tras los Pactos Lateranenses en 1929, hicieron parte de Italia una especial y singular relación con el Papado y la Santa Sede. Es algo que se nota, se vive y se asume por la misma sociedad italiana de manera imperceptible en el conjunto de las actividades, eventos y acontecimientos que se dan mutuamente. La expresión religiosa que en general se mantiene en todos los estamentos sociales respecto al Romano Pontífice, y a la Iglesia en particular es muy evidente; se puede decir que es de exquisito respeto que llega hasta los más mínimos detalles, de cortesía, amabilidad y delicadeza por parte de las autoridades y el pueblo de Italia. No se puede olvidar que parte de ese territorio había formado parte de manera secular de los territorios pontificios bajo la soberanía temporal del Papa de Roma. La capital era la ciudad de Pedro, donde fue martirizado; Roma era, es y será siempre la diócesis del Santo Padre. La Ciudad Eterna manifiesta, expresa y concita la universalidad de la Iglesia. Esta Iglesia, que aparecía como una sociedad independiente y que era autónoma, que se manifestaba por medio de la Sede Apostólica y que necesitaba tener una soberanía propia para ejercer su actividad misionera, profética y evangélica sobre todo el mundo, sustentada en un Estado: el Estado de la Ciudad del Vaticano.

\section{CONCLUSIÓN}

Para terminar hemos querido establecer claramente lo que entendemos por Protocolo y sus posibles definiciones. Nos hemos adentrado en las diversas posturas y opiniones sobre el tema para tratar de adecuar nuestras intenciones a lo que se puede entender, y de hecho se entiende por el Protocolo en general, las distintas concepciones sobre el término que se manejan coloquialmente y las acepciones que presentan en la vida social el Protocolo y sus clases. A continuación hemos expuesto lo que aceptamos y comprendemos desde lo que es la Iglesia, la Santa Sede y el Estado de la Ciudad del Vaticano. Como hemos adelantado los tres conceptos son diferentes aunque muchas veces en la práctica los tomamos como sinónimos y no lo son. Es

50 Vid. Martina, G., La Iglesia de Lutero a nuestros días. Vol. IV. Época del Totalitarismo, Madrid 1974, págs. 187-191. 
más, la gente en general los confunde o simplemente no hace acepción a los conceptos de cada uno.

La Iglesia es la comunidad de los creyentes en Cristo o también Pueblo de Dios; la Santa Sede o Sede Apostólica es la acción del Sumo Pontífice en cuanto Vicario de Cristo, mediante la cual se administra la Iglesia y es la máxima expresión de su autoridad, en cuanto al gobierno de la propia Iglesia; y el Estado de la Ciudad del Vaticano es la soberanía del Santo Padre, que se ejerce mediante los poderes temporales y le sirve para mantener su plena independencia jurídica, para que nadie pueda influir o someter a su imperio la acción evangelizadora que desempeña el Papa y que está por encima de cualquier poder temporal de este mundo. Seguimos adelante para desarrollar los protocolos que existen en los tres ámbitos descritos. La liturgia como Protocolo de la Iglesia, en todas sus acepciones y manifestaciones donde pesan fundamentalmente la acción de la tradición y las costumbres inveteradas. La liturgia con todo su esplendor, belleza y riqueza visual que nos lleva a vivir la dimensión transcendente del hombre y nos transporta a otra dimensión espiritual y divina. El Protocolo seguido por la Santa Sede, en todas sus manifestaciones referentes a los acciones del Romano Pontífice en las celebraciones y ceremonias en cuanto Obispo de Roma y sucesor de Pedro en el Colegio Apostólico; se incluyen todos los actos que realiza la Secretaria de Estado y en general toda la Curia Romana. De ahí, que la genuina liturgia recoja toda la expresión de los gestos y de las palabras que se pronuncian, y que contienen ampliamente el sentido humano y la conexión con la realidad sobrenatural; que el hombre que peregrina en el camino de la vida busca ante todo la Verdad, verdad que también aparece bajo el velo de la Belleza y de lo Bueno. Por último llegamos al Protocolo seguido en el Estado de la Ciudad del Vaticano. Éstas se encuentran en relación con los actos protocolarios de cualquier Estado, y por tanto, aplicables al propio Estado Ciudad del Vaticano. Llevan la referencia a las Visitas de los Jefes de Estado y de Gobierno al Vaticano, la presentación de las Cartas Credenciales de todos aquellos países que mantienen relaciones diplomáticas con la Santa Sede, mediante Embajadores Plenipotenciarios, que son acreditados formalmente. El Protocolo debido al óbito del Santo Padre; la celebración de los solemnes funerales y el sepelio; la elección del nuevo Papa con sus plenas y actuales tradiciones y la Misa de inicio del nuevo pontificado con la llegada de las máximas autoridades políticas, sociales, culturales y religiosas de todo el mundo y el saludo oficial y cordial del Santo Padre a todas las delegaciones oficiales, finalizada la celebración, en el interior de la Basílica de san Pedro. Por 
último poner de relieve la relación especial de la Santa Sede por medio del Estado de la Ciudad del Vaticano con Italia por las especiales concurrencias históricas entre ambas entidades, resaltando la positiva actitud del pueblo italiano.

Por eso la personalidad de carácter internacional de la Santa Sede está orientada al orden de la soberanía espiritual mientras que la personalidad internacional del Estado de la Ciudad del Vaticano, se encuentra en orden a la soberanía político temporal. Ambas entidades sirven a la Iglesia para su actuación libre en todo el orbe para seguir llevando el anuncio del Evangelio. El Estado de la Ciudad del Vaticano está en función de la Sede Apostólica o Santa Sede y ésta es el soporte con personalidad jurídica. La primera no es imprescindible para la existencia de la segunda, pero sí necesaria para su normal desenvolvimiento temporal con plena libertad. Su finalidad es, ante todo, garantizar la independencia del Romano Pontífice en el ejercicio de su labor como Pastor de la Iglesia y evitar cualquier pretensión del poder temporal de inmiscuirse en dicha misión pastoral. No podemos olvidar, en este sentido, la condición del Santo Padre como Jefe de Estado, lo que le hace más accesible la visita a lugares y territorios que podían ser más difíciles si fuese sólo como Cabeza de la Iglesia católica.

Con relación a la naturaleza jurídica del Estado de la Ciudad del Vaticano, la doctrina se basa en la misma finalidad para la que se creó dicho Estado. Es la base de índole territorial que sirve a la Sede Apostólica. Por eso es importante que el protocolo, referente a los temas eclesiásticos, especialmente al mundo católico, sea cada día más imprescindible para cualquier persona que se acerca al hecho religioso debido a su incidencia en los demás tipos de protocolo concernientes a la esfera civil. 
